\title{
Biospheric Energization
}

Budding $E^{*}$, Gündüz G, Ozel ME and Demircan $O$

Astrobiology Group, Çanakkale Onsekiz Mart University Çanakkale, TR 17020, Turkey

\begin{abstract}
We discuss a physical model relating to the origin and development of lifelike processes, paying particular attention to the continual growth of systemic energization and complexity from very simple beginnings. To approach recognizable life forms in the terrestrial case, we refer to a molecular ('ABC') process consisting of the following elements: A -- a molecular structure that can temporarily stores a local energy excess; B -- a catalyst that, making use of the local energy excess, catalyses also itself, and comes in a small number of slightly different forms involving different reaction steps; $\mathrm{C}$-- a disposable tool molecule that assists and tunes the response of the $\mathrm{A}$ and $\mathrm{B}$ molecules to the (time-dependent) local source of energy. This local source of energy is external to the ABC system, and we take it to be solar photons (or a comparable flux from a solar-like star).

We show that the system can evolve from very simple beginnings to a progressively more highly tuned, energized and complex responding biosphere, that grows exponentially; albeit with a very low net growth factor. We note the inherent instability suggested by this can be related to the "low L" solution of the Drake equation. We consider possibilities for similar processes in the outer regions of other planets of the solar system or elsewhere. We outline some possible observational checks for the principles of this model.
\end{abstract}

Keywords: Biosphere; Energization; Star; Boltzmann; Solar flux

\section{Definition of Life}

NASA has proposed that life be taken as a self-sustained chemical system capable of undergoing Darwinian evolution. This definition can be found consistent with general physical principles. The life chemistry encountered on Earth should accord with these principles, but as one particular case. This viewpoint allows us to seek for life in more generalized environments, although in this article we confine attention to planets and possible living systems having understandable comparisons to the terrestrial example. Further discussion of this and related matters was given by Budding et al. [1].

\section{Basic Physics and Life}

Budding et al. [1] contrasted the energization of locomotion in classical mechanics with that of a living system, noting the distinguishing role of metabolism, which implies a continuous supply of power directed specifically to maintain living [2]. Metabolism's highly ordered energization system essentially involves that field of chemistry called biochemistry, and it is within the spirit of Darwinism to regard the latter as having evolved from the former. We may thus regard life as a self-sustained and evolving chemical system, but reducing this selfsustenance and evolutionary character to physical terms calls for close consideration.

The least action principle of classical mechanics has parallels with the probability maximization underlying Boltzmann's distribution of the energies of an assembly of particles in statistical mechanics in that they both involve mathematical extreme. Boltzmann's distribution refers to a temperature $T_{E}$, which, in turn, implies a surrounding environment and associated radiation field. The argument is direct so long as $T_{E}$ can be specified, even if the assembly requires some response (or 'relaxation') time $\tau_{R}$ for the distribution to be attained. It becomes less direct if the environment's temperature is allowed to vary, on a timescale $\tau_{E}$, say. If $\tau_{R}<<\tau_{E}$ then we can expect a Boltzmann distribution for any given $\mathrm{T}_{\mathrm{E}}$. We could also expect a comparable distribution when $\tau_{R} \gg \tau_{E}$, if there is some average $\left\langle\mathrm{T}_{\mathrm{E}}\right\rangle$ that applies to the long timescale $\tau_{R}$. It becomes more complex if, for some system, is of the same order as $\tau_{E}$. A quasi-resonant configuration can be anticipated by moving to the frequency domain $v(=1 / \tau)$, with $v_{R} \sim v_{E}$. In particular, if $v_{E} \approx 2 v_{R}$ the possibility of driven or parametric resonance occurs: the responsive system becoming progressively more energized from the cyclic driving variation affecting the environment. This idea strongly influences our approach. We visualize a steady progression from a simple, low Q-factor (energy stored/energy lost per cycle), fairly uniformly dispersed, and marginally 'disequilibrium' chemical activity to an ever more stringently defined, highly differentiated, complex and hierarchically structured biochemical matrix.

This possibility is not overt in Boltzmann's [3] view of a predominating universal tendency towards random disorder ('heat'), in line with the second law of thermodynamics. While sensing the disparity between this tendency and the existence of living systems that grow, evolve and become more complex, Boltzmann and others have assumed that the solar flux can drive the biosphere, rather in the way that the potential energy gradient from an inland water head drives a (not necessarily simple or static) river system. Boltzmann then regarded Darwinian evolution as a competition for entropy within living systems. Entropy, in the sense of number of available states, or information required to cover the essential properties of a system, may well increase in evolving life-forms. However, such forms are not consistent with the specific probabilities for the arrangements that would be found in thermal equilibrium (e.g. a Boltzmannian distribution of particle energies). A satellite picture of the Earth at night, for instance, readily reveals the considerable excess of emitted high-energy photons above what would correspond to the night-time temperature of the planet.

*Corresponding author: Budding E, Astrobiology Group, Çanakkale Onsekiz Mart University Çanakkale, TR 17020, Turkey, Tel: 00644232 6388; E-mail: ed.budding@gmail.com

Received January 30, 2015; Accepted February 20, 2015; Published February 28, 2015

Citation: Budding E, Gündüz G, Ozel ME, Demircan O (2015) Biospheric Energization. Astrobiol Outreach 3: 128. doi:10.4172/2332-2519.1000128

Copyright: @ 2015 Budding E, et al. This is an open-access article distributed under the terms of the Creative Commons Attribution License, which permits unrestricted use, distribution, and reproduction in any medium, provided the original author and source are credited. 
Schrödinger [4] probed further into this issue, paying particular attention to the problem of "order from disorder". Schrödinger interpreted this as a local absence of equilibrium (i.e. disequilibrium) of the living subsystem: the measure of disorder could be reduced in this subsystem at the expense of an increase in that of the surroundings overall. Within an enclosed system the continuation of such disequilibrium contravenes thermodynamical understanding. However, if the containing environment is unbounded ('open') the overall entropy may still increase, while a subsystem becomes more ordered. While the maintenance of thermal disequilibrium in the biosphere thus necessitates an open system, lack of closure of the overall system has still not been shown sufficient to generate and progress living forms to the degree observed and in the time available.

More recently, a distinction has been drawn between increasing disorder and entropy. When a disequilibrium substructure occurs, ordered processes can be set up that work against the energy gradients producing the disequilibrium more effectively than in a randomly disorganized configuration. Such processes have been termed "dissipative structures" [5], and their action consistent with a maximal "energy degradation" [6]. This approach anticipates lifelike processes developing in any driven disequilibrium in a general way, while not yet specifying the driving mechanisms or any cycling (periodic) interactions that may be found in particular identifications of life.

Living systems, while generally composed of a large assembly of molecular particles ${ }^{1}$, are usually recognized, biologically, as individuals, having a high degree of ordered self-containment. Biologists often confine attention, regarding what is agreed to be fully alive, to such ordered structures, either mono-cellular or groups of cells with sufficiently strong interactions to constitute a multi-cellular organism. But, although life on Earth has evolved to this highly differentiated condition, in which cell-based life commands center stage, there are reasons to take an integrated or 'biospheric' view.

A natural place to look for the origin of the power driving the terrestrial biosphere is the Sun. The solar flux amounts to around 1.36 $\mathrm{kW}$ for each projected square metre above the Earth's atmosphere. The total incident power over the whole Earth is then around $2 \times 10^{14}$ $\mathrm{kW}$ or $\sim 7 \times 10^{21} \mathrm{~J}$ per day. There may be other energy sources, but the location of living matter about the Earth and the central role of photosynthesis within biology [7] push solar flux as the most likely main driver. A reasonable estimate for the total energy driving the entire biosphere is $\sim 10^{12} \mathrm{~kW}$ [8]. The solar flux thus has enough power to drive this 'engine': the problem is about how it works. Life is not simply a dissipative mechanical system like the weather: it evolves to greater complexity while exhibiting Darwinian self-sustenance, whose nature invites clarification.

\section{Chemical Potential Energy of the Biosphere}

We can consider the interplay of potential and kinetic energies also at the microscopic level of the arrangements of charged particles within atoms or molecules. 'Chemical potential energy' (CPE) then refers to the electrostatic potential associated with the relative excitation levels of valence electrons. Chemical interactions are mostly accounted for by the changing configurations, or energy states, of such electrons. If, as a result of some anisotropy, exothermic reactions occur, particulate potential energy tends to diffuse irreversibly into electromagnetic radiation. An endothermic process implies an atypical initial configuration within a given environmental temperature, which

\footnotetext{
1 The term molecule in this article denotes a very small particle, i.e. a somewhat looser usage than would normally apply to a quantified chemical reaction.
}

becomes regularized into a more probable arrangement. In either case, physical results are comparable to the degradation of macroscopic motions [1]. In normal conditions, naturally occurring processes tend toward configurations whose typical valence electron energies are comparable to those obtaining randomly at the ambient temperature of the local environment, i.e. a Boltzmann distribution.

But this is not the case in the surface regions of planet Earth! There is a large residual CPE among the reagents of the biosphere, whose existence can be seen as the key physical problem posed by life. This energy can be estimated to be of the order of $10^{23} \mathrm{~J}$ in total. This begins to compare with the total thermal energy of the Earth's atmosphere of $\sim 10^{24} \mathrm{~J}$; that is $\sim 1.5 \times 10^{5} \mathrm{~J} \mathrm{~kg}^{-1}$, while the average energy density of biotic matter is now some $3 \times 10^{7} \mathrm{~J} \mathrm{~kg}^{-1}[9,10]$. The physical significance of the ratio of these two energy densities $(\sim 200)$ was pointed out by [1].

A similar point arises from the existence of $3.71 \times 10^{19}$ moles of free oxygen in the Earth's atmosphere, that can be compared with a more feasible initial planetary atmosphere made up of lower chemical potential (more tightly bound) molecular arrangements, such as carbon dioxide, water, perhaps methane, or other constituents. Methaneoxygen combustion yields some $572.2 \mathrm{~J}$ per mole [11], so that a reverse process of oxygen liberation can be interpreted as a net potential gain of $\sim 2 \times 10^{23} \mathrm{~J}[1]$. The very significant approximate equality of this datum to the potential energy yield of all organic matter around the Earth's surface was pointed out already a long time ago by Haldane [12], and it was reiterated in ref [13] account of the biosphere's energization.

Typical chemical reactions that might occur naturally near the temperature and pressures about the Earth's surface involve such short interaction times that local excess molecular energies should be swiftly radiated away or lost through kinetic collisions with other molecules, until approximate equilibrium with the thermal energy of the surroundings is achieved [14]. Life on earth is thus generally recognized to be distant from the thermal equilibrium of standard thermodynamics [15]. But we can reasonably assume that the excess $\mathrm{CPE}$ of this disequilibrium came from the solar influx converted and retained by living forms: if not, then from where?

\section{Homeostasis, Restoration and Darwinian Self- sustenance}

A characteristic property of a living system is its homeostasis: a restorative behaviour 'attracted to' continued living [16,17]. This attraction is strongly related to its Darwinian character or 'aim to survive'. Most explanations of biological behaviour refer either directly or indirectly to this property; through its role in cell metabolism or implications for evolution. We focus now on the essential nature of the restorative mechanism underlying homeostasis.

Budding et al. [1] background this point in more detail. They consider, as a general case of restoration, the Sturm-Liouville type second-order differential equation

$$
D^{2}\{y\}+\lambda(\mathrm{t}) \mathrm{D}\{\mathrm{y}\}+\omega^{2}(t) y=0
$$

(time derivatives denoted here by $\mathrm{D}\{\}$ ); known as the parametric oscillator equation. The left members of this equation include first the accelerative term in $\mathrm{D}^{2}$, followed by the dissipative term $\lambda(t) D$. The oscillatory coefficient $\omega^{2}(t)$, with mean value $\omega_{0}$, includes also a driving component with mean frequency $\Omega_{0} / 2 \pi$, that can cause a steadily increasing energy of motion for suitable values of period and amplitude. 
The responsive oscillation y, satisfying Eqn (1), has an amplitude $\mathbf{y}_{0}$ that depends exponentially on the time $t$, i.e.

$$
y_{0}(t)=y_{01} e^{(\alpha-\mu) t}
$$

That grows or declines according as the stimulation coefficient a exceeds that of the dissipation $\mu$. The exponential character of this energization should be noted, even if the growth rate may be very small. The stimulation $a$ depends on the basic driving frequency. The latter can be taken to be $\sim 1 \mathrm{day}^{-1}$ for the insolation, but in our scheme there are actually two processes separated by $\sim 0.5 \mathrm{~d}$, so parametric driving could occur if the system's natural mean response time was about a day, and having an initial energy amplitude of the same order as the external driving function $\mathrm{f}_{0}$. The dissipation $\mu$ depends on the integrated effect of the damping coefficient ( $\lambda$ in Eqn 1 ), which in turn relates to the available behaviour of the system, i.e. at what rate it loses potential energy.

If we suppose that the CPE of a chemically responsive system, that can be regarded as a parametric oscillator subject to a daily solar energy input $\mathrm{f}(\mathrm{t})$, scales with the amplitude $\mathrm{y}_{0}(\mathrm{t})$ as above, then the current average CPE excess of biotic matter ( 200, see Section 3$)$ implies that $10^{12}(\alpha-\mu) \approx \ln 200$, or the mean value of $(\alpha-\mu) \approx 5 \times 10^{-12}$ day $^{-1}$.

Consider now the growth of oxygen in the Earth's atmosphere $[18,19]$ from the Earth's formation through to the present. The average slope for the logarithmic growth can be worked out as $\sim(4.30 / 4.8 \mathrm{By})$, which when translated into the exponential growth rate per day amounts to $\sim 5 \times 10^{-12}$ : essentially the same as the foregoing estimate. The same agreement is reflected in the comparability of the CPE of the biosphere regarded (a) as its presently combustible total energy, and (b) the energy required to liberate the mass of free oxygen in the Earth's present atmosphere from likely initial constituents noted in the preceding section. The liberation of bonded oxygen, with its implication of CPE enhancement, gives direct support for our resonating oscillator model of biosphere formation and development.

The selective quality built into the solution (2) can also be seen. Different chemical pathways can be associated with different values of the net energization coefficient $(\alpha-\mu)$. If such differences correspond to alternative possibilities for the same components in the same location and subject to the same dissipative effects, then the pathway that locally maximizes $(\alpha-\mu)$ will predominate there, due to its energy advantage. This interpretation physically parallels the Darwinian view of an advantaged mode having an enhanced chance of survival ('natural selection') $)^{2}$.

${ }_{2}$ A physics-based approach to the origin-of-life problem published by England (2013) recapitulates the point made about growth in the foregoing model (cf. Budding et al., 2012). Referring to England's (2013) statistical physics of a selfreplicating population of individual life forms, the population is set as $n$ at time $t$. The population being given, the probability $p(n)_{t}$ is here 1 . At time $t+\Delta t$, when the population is $n+\Delta n$ the probability that the number is $n$ has now changed by $-\Delta q$ from 1 , since $\Delta n$ is generally non-zero. The probability $p$ must always decline from certainty, in this context, $(\Delta p<0)$ but the change of population can be either positive or negative $(\Delta n>$ or $<0)$.

England's (2013) formula (9) recognizes that this probability depends on the number $n$ itself, and introduces two other terms $g$ and $\delta$, corresponding to given or adopted rates of growth and decay, respectively, to handle the variation of $\Delta n$. England's formula gives, in effect,

$\Delta p / \Delta t=-\Delta q / \Delta t=-g n\left\{\Delta q_{\mathrm{b}} / \Delta n\right\}+\delta n\left\{\Delta q_{\mathrm{f}} / \Delta n\right\}$

where $\Delta q$ and $\Delta q$, correspond to backward and forward displacements from the situation at time $t$, to a time when the population number has changed by $\Delta n$. When $n$ becomes large, the increments small and the behaviour of the population changes smooth, we can reasonably consider the changes $-\Delta q_{b}$ and $-\Delta q$ to be numerically the same as the change $\Delta p$ over the increment $\Delta t$. We can then cancel out the equal factors, and write

\section{Molecular Mechanism}

Budding et al. [1] sketch a molecular model that can behave as a parametric oscillator according to the foregoing, with references to relevant early-life studies that may substantiate their scheme experimentally. Here, we summarize the main points. The biochemical model for a circadian bacterial oscillator of Pavlidis and Kauzmann ([20] hereafter PK) can be compared with this scheme, although ours is directed in the first instance to a prebiotic ensemble of relatively simple molecules, from which a continually developing and recognizably Earth-like, biosphere evolves. Other evidence on the fundamental role of rhythmicity in biological systems was cited by Budding et al. [1]

Numerous alternative pathways and branchings may occur in relevant biochemical reactions. This issue was discussed by King [21] with a schematization similar to the one of Budding et al. [1]. King gave a necessary condition on the ratio of side-reactions to promoting reactions that should be satisfied in the milieu in which an autocatalytic sequence could be maintained. While such schemes tend to be directed initially towards originating a sugar-base combination comparable to a simple nucleoside, they could apply at different levels. With this in mind, the 'ABC' formulation which follows should be regarded as having a wider application than might hold for specific molecular combinations. The underlying issue is one of suitably timed molecular binding-energy decreases (potential energy increases) that make available work used to support or build structures that enhance stability of the autocatalysis, with feedback in the interactions of mixed chemical systems a key element. The first stage in Budding et al. scheme is an endothermic reaction sequence, induced by the solar energy flux $f$, in the presence of a catalyst (B). In the terrestrial case, it should have originated from some very simple proto-carbohydrate, such as formaldehyde $\left(\mathrm{CH}_{2} \mathrm{O}\right)$, that would engender more complex subsequent reactions [22-24]. The main outcome is a molecule deriving from an oxygen reducing process with enhanced CPE that is stable over the timescale of at least a halfday. This end product ' $A$ ' is then a photosynthetically produced sugar: probably, but not necessarily, along the lines considered by Garrison et al. $[25,26]$. This 'A-subprocess' we write as:

$$
\left[\mathrm{CO}_{2}+\mathrm{H}_{2} \mathrm{O}\right]_{\mathrm{n}} \underset{f,[B]}{\longrightarrow} \mathrm{A}+n \mathrm{O}_{2} \text {, }
$$

Where $\mathrm{n}$ is an appropriate small integer. The (organic) molecule A can be regarded as a temporary energy source and driver of subsequent molecular interactions. Pertinent to the origin of modern life forms was the discovery by Powner et al. [27] of a pathway to the nucleoside ribo-cytosine involving, as a key reactant, 2 amino-oxazole. Figure 1 of Powner et al. [27] shows this latter molecule in an A type subprocess, in which glyceraldehyde $\left(\mathrm{C}_{3} \mathrm{H}_{6} \mathrm{O}_{3}\right)$ is catalysed to arabinose $\left(\mathrm{C}_{5} \mathrm{H}_{10} \mathrm{O}_{5}\right)$. Nucleosidic sugar + base assembly then follow, even if not by the more direct pathway supposed by early experimenters. We indicate in (I) the key catalytic role of the second conceptual basic molecule by B: the brackets around it are meant to suggest that other molecules may affect its action. Continuity of the A-B cycle is achieved during the hours of darkness, when the A type molecule is involved in a mildly exothermic (energy release $\phi$ is less than $\mathrm{f}$ absorbed in the A process) reproduction of the B type molecule in the 'B-subprocess'.

$$
\begin{aligned}
& \Delta n=(g-d) n \Delta t, \\
& \text { or, by integration, } \\
& \quad n=n_{0} \exp \{g-d\} t,
\end{aligned}
$$

where $n_{0}$ is the size of the population at an initial time.

But (3a) is the same as Equation (2) above, supporting the relevance of the driven parametric oscillator model of biospheric energization to the growth of biospheric life forms, within the thermodynamic argument of England [22] 


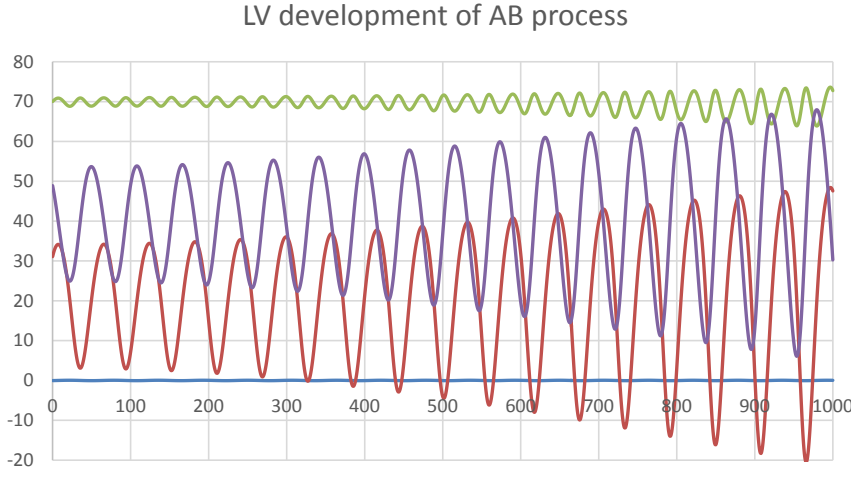

Figure 1: Numerical integration of the modified LV equations (7-8). Axes have arbitrary units.

Evolved B type molecules on the Earth could be expected to include ring structures (as A), and, keeping in mind the amino basis of enzymes, involve nitrogen [28-30]. PK denote this catalyst by E, with an excited state as $\mathrm{E}^{+}$, identified as adenosine diphosphate (ADP). The PK modelling underlines the intimate relationship of the two components $\mathrm{A}$ and $\mathrm{B}$, condensing to the compound $\mathrm{AB}$ at the center of the sequence and controlling its outcome.

Using the formalism of the previous section, we schematize the A subprocess in the form

$$
\mathrm{D}\{\mathrm{A}\}=\mathrm{k}_{1} \mathrm{~B}+\mathrm{f}_{1}(\mathrm{t}),
$$

where $\mathrm{D}\{\mathrm{A}\}$ denotes the rate of sugar molecule formation, in dependence on the local density of B type catalysts, but with dependence on the variable solar insolation, mainly through the environmental function (e.g. local temperature) embodied in the term $\mathrm{f}_{1}(\mathrm{t})^{3}$. In practice, the coefficient $\mathrm{k}_{1}$ would also be, to some extent, timedependent. Within the present highly simplified context, we expect other environmental factors to remain essentially constant.

Powner et al (2009) note the primary role of urea as a source of the $\mathrm{B}$ type component in their nucleoside generation model. With this in mind, we can write for a B-subprocess:

$$
\left[\mathrm{AB}+2 \mathrm{NH}_{4} \mathrm{OH}\right]_{\mathrm{n}} \stackrel{\phi}{\longrightarrow} 2 \mathrm{~B}+n \mathrm{H}_{2} \mathrm{O}
$$

In this very simple approach, $\mathrm{B}$ is a derivative of the sugar-amine condensation in an ammonia solution. In a similar way to Eqn (3) we can write for this B subprocess

$$
\Omega \text {, }
$$

Where $\mathrm{A}$ indicates the amount of intermediate $\mathrm{AB}$ compound (=EX in PK), for which we write

$$
\mathrm{A}=k_{4}(A+B)
$$

If we differentiate the first relation again and substitute from the one just given we find

$$
\left.D^{2} A\right\}=-\omega^{2} A-\lambda D\{A\}+f_{2}(t),
$$

With $\omega^{2}=k_{1}\left(k_{3} k_{4}-k_{2}\right), \lambda=k_{3} k_{4}$ and $f_{2}(t)$ is the oscillatory function $f_{1}^{\prime}+k_{3} k_{4} f_{1}$, together with other small contributions arising from time derivatives of the coefficients $\mathrm{k}_{i}$. Eqn(5) has the parametric oscillator form, yielding a consequent exponential growth in the appearance of sugar type molecules, if $\alpha>\mu$.

${ }^{3}$ Note here that $f_{1}$ contains a suitable coefficient ensuring that Eqn (3)'s units - i.e. those of the local concentration rate - balance.
In a similar way we can show that B satisfies the similar form

$$
D^{2}\{B\}=-\omega^{2} \mathrm{~B}-\lambda D\{B\}+f_{3}(t),
$$

With $f_{3}=\left(k_{2}-k_{3} k_{4}\right) f_{1}$. Since we expect oxygen to be liberated as a byproduct of the A-B process it too shows a similar steady exponential growth, and, by implication, the chemical potential energy of the process as a whole.

A key point is that the natural timescales $\left(2 \pi / \omega_{0}\right)$ for both $A$ and $B$ subprocesses should be of about a day in typical terrestrial conditions. Such timescales, demonstrated by Weber [30] for feasible real molecules, are relevant to the susceptibility to diurnal resonance for the whole scheme. The intermediate $\mathrm{AB}$ compound has an important regulatory role. The A-B process overall is controlled, multiplicative and CPE-enhancing, thus showing elementary lifelike properties.

The simple reactions sketched as (I) and (II) above point to biochemistry evolving from a simpler chemistry in a terrestrial environment, with formative roles for the quasi-organic (i.e. naturally occurring) molecules formaldehyde and urea.

Budding et al. [1].took the original A-subprocess to be natural photosynthesis, following the idea of Garrison et al. [25]. Adopting some order-of-magnitude parameter values, they derived a natural oceanic daily oxygen yield of $\sim 10^{9} \mathrm{gm}$, which would act as a seed for the subsequent gradual atmospheric build-up. The present atmospheric proportion of $\sim 21 \%$ points to an advanced stage of the terrestrial biosphere.

\section{Lotka-Volterra Formulation}

The oscillatory behaviour coming from Eqns (5) and (6) can be reconciled with a Lotka-Volterra (LV)-like formulation of the system, albeit with some small modification. Let us write

$$
\begin{aligned}
& D\{\alpha\}=\kappa_{1} \beta+f_{1}(t)-\kappa_{3} \alpha \beta, \\
& D\{\beta\}=\kappa_{2} \alpha \beta-\kappa_{4} \alpha,
\end{aligned}
$$

Eqn (7) accounts for a numerical proportion $\alpha$ of free A type molecules in a local environment, as in the A-subprocess, regarding this as dependent on the proportion of catalytic B type molecules $\beta$, as well as some (oscillatory) external power source (driving function) $\mathrm{f}_{1}$, while the $A B$ compound has sequestered free A types that would otherwise be available for such condensation, to an extent that depends on the product $\alpha \beta$. In a similar way, Eqn (8), parallels the B subprocess, where B type molecules are liberated from their AB-type source, but may then be removed from the milieu by association with ambient free A types. Eqns (7) and (8) are complementary to the standard LV form, normally written (without the driving term) as

$$
\begin{aligned}
& D\{\alpha\}=\kappa_{1} \alpha-\kappa_{3} \alpha \beta, \\
& D\{\beta\}=\kappa_{2} \alpha \beta-\kappa_{4} \beta,
\end{aligned}
$$

But it can be shown that the "collaborative" forms (7) and (8) share similar oscillatory behaviour to the "competitive" relations (7a) and (8a).

In Figure 1 we show the results of a numerical integration of the system (7)-(8), against linearly scaled (but by arbitrary amounts) axes. The lowest curve follows the changes of $\alpha$ (A type molecules), and the middle curve the complementary changes of $\beta$ (B types). In terms of the original [1], scenario, the peaks of A-type production could be expected around noon, or in the early afternoon. B type maximization has occurred by the preceding early morning hours. The highest 
curve follows the more constrained variation of $A B$ type molecule development, having twice the frequency of its progenitor molecules.

Predator-prey interactions have been presented more in terms of stochastic encounters by Maynard Smith [31], and the corresponding $\mathrm{AB}$ synthesis problem can be so formulated.

Figure 2 shows an example of such interactions where the diurnal input power term appears as the large amplitude blue sine wave at the bottom. The upper red curve corresponds to A-type molecule concentration, with $\mathrm{B}$-types appearing as the green curve. The $\mathrm{AB}$ molecular density shows up in purple in this diagram. The longer-term exponent indicates the steady growth in the numbers of such molecules.

\section{Growth, Evolution and Complexity}

The A-B cycle thus acts as a parametric oscillator in a near-resonant condition with the diurnal rotation and molecular condensation timescales. During the day, B catalyses the production of more A molecules from initial constituents. Towards night, A-type molecules condense with the $B$ type and at lower nocturnal temperatures this leads, via the $\mathrm{AB}$ intermediary, to the formation of more $\mathrm{B}$ molecules [20]. Sometimes a somewhat different molecule B' may be involved. The possibility of rival catalysts $B$ and $B$ ', or control compounds $A B$ or $A B$ ', institutes molecular evolution. This is on the basis of preferential selection in a given environment depending on efficiency of the resonance (B or B') for CPE generation, even at this fundamental level [32]. This simple AB or AB' dichotomy has an analogue in the 'black' or 'white' conceptualization of Daisyworld [33]. Early biospheres on planets with moderately uniform topology could thus be expected to show a zonal structure. Zonal propagation of the energization may also be approachable via the Telegrapher Equation in spherical polar coordinates, but we consider here other issues arising with the developing energization.

If driving exceeds dissipation, the process must grow, but not simply in linear amplitude of excitation or numbers of A and B molecules. The available modes of carbon-based molecular chemistry allow for the formation of more complicated, higher $\mathrm{CPE}$, structures. With ongoing driving, the catalytic function becomes not just from single molecules, but groups of more complex, finely tuned, molecular sequences, genes, or macrostructures, co-operating towards the resonance-driven $\mathrm{CPE}$ enhancement. There should then be not just one, but a spectrum of A-B cycles, filling in the available bandwidth for which $\alpha>\mu$.

But how does controlled organization develop? It is the $A B$

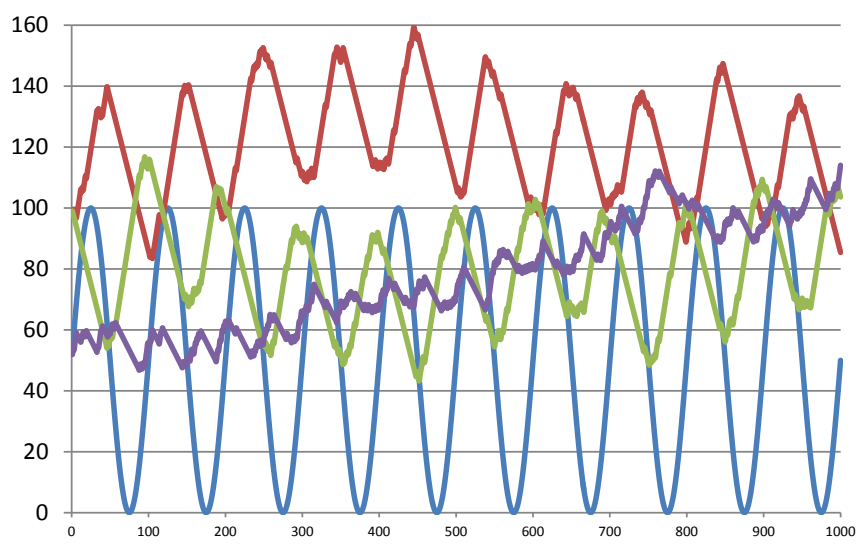

Figure 2: Stochastic interactions of $A$ and $B$ type molecules. compound that promotes survival of the surrounding protobiotic molecular ensemble through its negative feedback. We indicated above that the B subprocess is associated with the release of energy $\phi$, but this is at a rate determined symbolically by the value of the quantity $k_{4}$. At this point, we encounter a third molecule - the $\mathbf{C}$ type - that chemically relates to the $\mathrm{AB}$ compound. In effect, reactions involving, or 'targetting' such $C$ types by the $A B$ (or $A B$ ') types influence the dissociation rate $\mathrm{k}_{4}$ in $(4 \mathrm{a})$. In turn, this affects the natural frequency $\omega$ and subsequent rate of multiplication and evolution, depending on the separation of from $\Omega$ or possibly submultiples of $\Omega$. C type molecules, coding optimal responses for the relevant local environmental conditions, can be regarded as proteins in the terrestrial case. They are outside the catalytic $A B A B$ ' protonucleotide sequence, but would be maintained through subsequent multiplication if promoting the underlying energizing resonance. $\mathrm{C}$ type molecular structures will naturally evolve an increasing role in regulating local conditions to promote internal homeostasis of surviving forms.

In due course, and with the steady increase of biospheric $\mathrm{CPE}, \mathrm{AB}$ type molecules themselves compound to more complex ABAB' forms, made possible by the versatility of suitably energized carbon bonding. This development can be expected around nodes in the environment, where advantageous circumstances for the growth of one mode $(\mathrm{AB}$, say) give way to another ( $\left.A B^{\prime}\right)$. The appearance of $A B A B$ ' forms at a node can recapitulate the same modified LV equations (7-8) as for the original $\mathrm{AB}$ combination, with $\alpha$ now standing for $\mathrm{AB}$ and $\beta$ for $\mathrm{AB}$ '. Note then the early and essential appearance of recapitulation as a characteristic of living processes.

Maintenance of the driving resonance implies that the mean daily $\mathrm{B}$ molecule production must be maintained by the overall ensemble, implying, at a fundamental level, the continued demise of individual $\mathrm{AB}$ types, i.e. the death of particular or individual forms. Genetic 'immortality' [34] might perhaps be countenanced in that a given driving function in a given environment will correspondingly reproduce, by the most advantaged pathway, essentially similar advantaged molecules.

$\mathrm{ABAB}^{\prime}$ (etc.) molecules retain more information about environmental variation, assisting multiplication and CPE increase, but in a more specifically defined local environment; the different links in an $\mathrm{ABAB}$ ' molecule having complementary regulating actions. The slow build-up of more complex $A B A B$ ' structures and corresponding $\mathrm{C}$ or $\mathrm{C}^{\prime}$ molecules, also deposits a proportion of the net CPE increase into fewer molecules, giving a proportionally large increase in the energization per molecule of longer protonucleotide chains. Surviving development, involving these more complex sequences, manifests as more organized behaviour, with highly energized long molecules imparting hierarchical structure into the control function.

Local conditions harmonizing with this increasing specification and control may tend to concentrate advantaged lifelike processes into lacunae containing local center's of organization. Rock or tidal pools, as well as clay molecular binding templates, have been surmised to provide an early natural realization of this scenario [35-37]. This relates to the feedback affecting the distribution of $\mathrm{A}, \mathrm{B}, \mathrm{AB}, \mathrm{ABAB}$ ' (etc., as well as corresponding $C$ types) within a lacuna. This control distribution can be expected to develop a pyramidical or 'sandpile' form [38], with characteristic slope $\gamma$ coming from the stability requirement that $\delta \mathrm{U} / \delta \mathrm{E} \leq \gamma$, where $\delta \mathrm{U}$ represents a mean fluctuation of local energy density (e.g. local temperature changes) and $\delta \mathrm{E}$ corresponds to the typical inter-link binding energy of an $A B A B^{\prime}$ type molecule. Numbers of corresponding $A B, A B A B$ ', $A B A B B^{\prime} A B$ " (etc.) molecules 
would then tend to decrease geometrically in proportion to $\gamma$, while the overall height of the pyramid (i.e. length of the longest molecule in the pile) increases in proportion to the general growth $(\alpha-\mu) t$.

On the basis of the observed number $\mathrm{n}$ of $\mathrm{AB}$ types, in a molecular chain containing $\mathrm{N}$ such pairs that may become indefinitely large, we find the number of permutations of the $\mathrm{AB}$ complexions $P_{n}^{N}=N ! /(N-n) !$. The complexity measure $\log \left[\log \mathrm{P}_{n}^{\mathrm{N}}\right]$, regarded as a function of time, using observed fossil data, can be seen to show a logarithmic character in its general growth [39]. Using the Stirling formula for $\log$ N!, we can confirm, since the argument of the complexity measure is itself a logarithm, that the general growth of the number of $\mathrm{AB}$ type molecules must itself be exponential, in order that the outer logarithm's argument be approximately linear with time. Budding et al. [1] argued, in this way, that the exponent of complexity growth derived from fossil evidence is generally consistent with the value inferred from the current energization of the biosphere derived in Section 4. More detailed features in the growth of complexity require further consideration of the particular circumstances of development.

We argued above that the restorative development in parametrically driven molecular ensembles implies that neighbouring particle combinations (in space and phase) other than those initially propelled would tend to take up the same local energization, provided they have the same, or very similar, properties. An interesting question is then this: at what stage does this 'bottom up' restorative development of the biosphere start to look more like 'top down' regulated replication of its infrastructures? Growing complexity of the latter seems likely increasingly to mask the underlying biospheric driver (cyclic insolation) as evolution proceeds: however, the fundamental role of biospherically generated global reagents, such as $\mathrm{O}_{2}$ or $\mathrm{CO}_{2}$, shows that lacunal life can never be entirely self-organized.

Do the organized molecular lacunae become cells? Cells would be a type of lacuna, in the way we have presented this organized energization scenario; but it is more useful to keep the lacuna concept that can pertain to a variety of levels separate from that of a cell, which has a much more clearly defined identity and function within biology. The rate of development of biospheric complexity indicates that cells have probably grown and evolved from the more primitive, but relatively large, viral proportion of the terrestrial biomass. In effect, these are energized $A B A B$ ' structures having particular $C$ or $C^{\prime}$ type molecules regulating their function sequence and natural timescale. The formation of collaborative substructures within a distribution of energized individuals to optimize net performance is a well-known issue in organization theory. It is reasonable to seek the origin of cells from virus-like precursor combinations as an example of this.

One single resonating A-B process can be conjectured to have become the predominant driver of early lifelike development on Earth. This is suggested by the single A type molecule (ribose) and the prevailing chirality of evolved biotic molecules, as well as a preference for the isotope $\mathrm{C}_{12}$ in living structures [40]. This same basic $A-B$ process may still be present within the ongoing biosphere, but the original molecular behaviour would by now have been considerably developed by secular evolution in a parallel way to the changes of atmospheric composition.

Recapitulation may help gain insights of this. The requirement for homeostasis within a surviving multicellular organism recapitulates that of individual cells. Stability of the biosphere recapitulates that within its lacunae. Surviving lacunae should thus interact collaboratively in general, though competitive episodes are clearly visible in nature. The exponential development of the biosphere is also echoed in
Haeckel's conjecture that 'ontogeny recapitulates philogeny'. The rate of phylogeny maps the logarithmic measure of biospheric complexity, so that time intervals for given increases in log (complexity) of species within the biosphere are proportional to their corresponding dates of first appearance. The complexity of an individual soma of an advanced species, meanwhile, should proceed linearly with time along its genetic (molecular) map during its own growth. Haeckel's conjecture implies that the early stages of this growth in complexity are telescoped into a relatively very short timescale.

\section{Observational Points}

A corollary to our growth model for biospheric energization is the implied long-term instability coming from the small, but positive, exponent in the solution of the underlying parametric oscillator equation. Although not identified as an inherent feature of the biosphere, the likelihood of its ultimate catastrophe has been referred to from various points of view [41]. The situation in which the lifetime $\mathrm{L}$ of an advanced technological civilization was essentially short (i.e. $\mathrm{L}<\sim 100$ y) was discussed by Bracewell [42]. In reviewing alternative scenarios of interstellar communications corresponding to different solutions of Drake's (1961) equation [43]. This alternative is the one most easily reconciled with Fermi's famous "where are they?" question. We do not detect "them", nor they us, because, cosmically, they (and ourselves) are not around long enough for any chance of meaningful dialogue.

Other observational implications of this energization model were presented by Budding et al. [1], who referred also to other planets in the solar system. New, high-precision exoplanet photometry may offer interesting opportunities in this respect. Close monitoring of the Jovian atmosphere shows that the Great Red Spot (GRS) must be dissimilar to general atmospheric cyclones that follow the formation and dissipation timescales of expectable meteorological effects (i.e. a few rotation periods). The GRS, on the other hand, actively propels itself against dissipative turbulence in its surrounding belt. The long-term survival of the GRS against this atmospheric turbulence can be estimated, from simple aerodynamic comparisons, to involve a power injection of order $10^{16} \mathrm{~W}$. The mean insolation over the GRS could be reckoned as of this order or somewhat greater: so that the Sun is able to drive the process energetically. The question of how this power might be transferred into organized restoration of this large-scale dissipative structure remains to be clarified, but the GRS is definitely a candidate for protobiotic energization. Excitingly, surface inhomogeneities comparable to the GRS but on the recently discovered 'Jupiters' of other stars may become photometrically detectable in the foreseeable future.

The discussion presented in this article is indicative and exploratory only. But we believe it provides a logical framework for consideration of the energization and progressive evolution of biospheres in a cosmic context, as well as an objective basis for experimental testing. It links together various fields of science and implies a multi-disciplinary approach. In this pursuit, we would encourage, and have hopes for, further broad international collaborations to help drive and coordinate functions.

\section{Acknowledgements}

The authors acknowledge support and helpful discussions from members of the COMU-based Astrobiology Group (comu-astrobiology@googlegroups.com)

\section{References}

1. Budding E, Aki C, Demircan O, Gündüz B, Gündüz G, Özel ME (2012) Turk.. J Phys. 36: 473-493. 
2. Harold FM (1986) The Vital Force: A Study of Bioenergetics. Freeman, New York.

3. Boltzmann L (1886) The Second Law of Thermodynamics, in McGuinnes B. (ed), (1974), Ludwig Boltzmann, Theoretical Physics and Philosophical Problems, D. Reidel, New York

4. Schrödinger E (1944) What is Life?, CUP, Cambridge.

5. Prigogine I (1955) Introduction to Thermodynamics of Irreversible Processes, Interscience Publ., New York.

6. Kay JJ (2000) Ecosystems as Self-organizing Holistic Systems: Narratives and the Second Law of Thermodynamics, in S E Jørgensen, F Müller (eds) Handbook of Ecosystem Theories and Management, CRC Press, Lewis Publishers, pp 135-160

7. Lieth $\mathrm{H}$ (1975) in Unifying Concepts in Ecology, W.H. van Dobben \& R.H. Lowe-McConnell, Dr W. Junk Publ. The Hague, 67-88.

8. Kondratyev KY, Krapivin VF, Savinykh VP, Varotsos CA (2004) Global Ecodynamics, Springer

9. Lieth H, Whittaker RH (1975) Primary productivity of the biosphere, SpringerVerlag, New York.

10. Smil V (1991) General Energetics: Energy in the Biosphere and Civilization, John Wiley.

11. Job G, Herrmann F (2006) European Journal of Physics 27: 353-371

12. Haldane JBS (1929) The Rationalist Annual 148: 3-10

13. Urey HC (1952) Proc. Natl. Acad. Sci. U.S.A 38: 351-363

14. Levine JS, Augustsson TR, Natarajan M (1982) Origins of Life 12: 245-249.

15. Nicolis G, Prigogine I (1977) Self Organization in Non-equilibrium Systems, Wiley.

16. Lovelock J (2003) Gaia: The living Earth, Nature 426: 769-770

17. Popa R (2004) Between Necessity and Probability: Searching for the Definition and Origin of Life, Springer.

18. Bekker A, Holland HD, Wang PL, Rumble D, Stein HJ (2004) Dating the Rise of Atmospheric Oxygen, Nature 427: 117-120

19. Kump LR (2008) The rise of atmospheric oxygen, Nature 451: 277-278.

20. Pavlidis T, Kauzmann W (1969) Arch. Biochem. Biophys 132: 338-348.

21. King GAM (1986) J Theor Biol 123: 493-498.
22. England J (2013) J Chem Phys 139: 121923.

23. Ricardo A, Carrigan MA, Olcott AN, Benner SA (2004) Borate Minerals Stabilize Ribose, Science 303: 196

24. Weiss AH, Krylov OV, Sakharov MM, Ghorochovatskii YB (2009) J. Food Processing, Preservation 2: 63

25. Garrison WM, Morrison DC, Hamilton JG, Benson AA, Calvin M (1951) Science 114: 416-418.

26. Löb W (1913) Über das Verhalten des Formamids unter der Wirkung der Stillen Entladung, Ber. Dtsch. Chem. Ges 46: 684.

27. Powner MW, Gerland B, Sutherland JD (2009) Nature 459: 239.

28. Hough I, Rogers AF (1956), J. Physiol. (London) 132: 28-29.

29. Oró J (1961) Nature 191: 1193-1194

30. Weber AL (2007) Origins Life Evol Biosph 37: 105-111.

31. Maynard Smith J (1972) On Evolution, EUP, Edinburgh 8-28.

32. Oparin Al (1957) The Origin of Life on Earth, Academic Press, New York.

33. Watson AJ, Lovelock JE (1983) Biological homeostasis of the global environment, Tellus 35: 284-289

34. Dawkins R (1976) The Selfish Gene, OUP, Oxford.

35. Bernal JD (1967) The Origin of Life, Weidenfeld \& Nicolson, London.

36. Fox SW (1995) Thermal synthesis of amino acids and the origin of life Geochim. Cosmochim. Acta 59: 1213-1214.

37. Walker SI, Grover MA, Hud NV (2012) Universal Sequence Replication, Reversible Polymerization and Early Functional Biopolymers: A Model for the Initiation of Prebiotic Sequence Evolution. PLoS ONE 7: e34166.

38. Bak P, Tang C, Wiesenfeld K (1987) Phys. Rev. Letters 59: 381-384.

39. Delsemme A (1998) Our Cosmic Origins, CUP, Cambridge.

40. Gleiser M, Walker SI (2008) Origins Life Evol. Biospheres 38: 293-315.

41. Rees M (2003) Our Final Century, Heinemann.

42. Bracewell RN (1974) in Intersteller Communications: Scientific Perspectives, ed C. Ponnamperuma and A.G.W. Cameron, Houghton Miflin Co., Boston, 102-116.

43. Tarter J (2006) Skeptical Inquirer Magazine, May issue. 